\title{
The VALTA project: Full scale conversion of CHP engine flue gas heat into electricity
}

\author{
Maurice-Xavier Francois', Kees de Blok ${ }^{2}$, Paul Bouakhao ${ }^{1}$, Manuel Niphon ${ }^{1}$,Jean Pierre \\ thermeau ${ }^{1}$, Bruno Guestin ${ }^{3}$, Luc Courtès ${ }^{3}$, Denis Clodic ${ }^{4}$ \\ ${ }^{1}$ HEKYOM, 2 rue jean Rostand, 91400- ORSAY, France mxfrancois@hekyom.com \\ ${ }^{2}$ ASTER, Smeestraat 11 NL8194LG VEESEEN, The Netherlands \\ ${ }^{3}$ SDMO, 12 bis rue de la Villeneuve, 29228 BREST, France, \\ ${ }^{4}$ EReIE, 3 rue de la Croix Martre, 91120 PALAISEAU, France, 12bis rue de la Villeneuve
}

Exhaust gas from combustion engines in commercial CHP systems or generator sets contains a significant amount of high grade thermal energy. Prior to exhausting this flue gas to open air, part of its thermal energy can be harvested by a thermoacoustic energy converter (TAEC) converting this heat, normally wasted or used for low grade heating, into electricity or cold (tri-generation).

The VALTA project originated in France and lead by HEKYOM aimed to design and build such a downstream energy converter to be applied to a commercial genset, converting $70 \mathrm{~kW}$ heat flue gas heat into $15 \mathrm{~kW}$ electricity. The equipment is supposed to convert exhaust gas enthalpy in the temperature range of $350^{\circ} \mathrm{C}$ to $200^{\circ} \mathrm{C}$ into acoustic energy and from there into electricity. The VALTA project was partially funded by ADEME, www.ademe.fr, in a specific program for "efficiency improving in industrial processes.

The VALTA project associates four Companies, HEKYOM and ASTER for thermoacoustics design and manufacture, SDMO for genset equipment implementation and EReIE for some contribution in heat transfer mechanisms by means of heat pipes

The concept of the VALTA heat recovery system comprises a few innovative embodiments.

One of them is the thermoacoustic geometry proposed by HEKYOM with 3 amplifiers positioned at small mutual distance and acoustically connected in series while thermally connected in parallel. Heat from the flue gas heat exchanger is transferred to the engine heat exchangers by dedicated looped heat pipe circuits. As compared with normal flow through heat exchangers this two-phase heat transfer mechanism significantly reduces temperature drop between the heat source and thermoacoustic process. It is known from Ceperley that thermoacoustic amplification process can be very efficient if the characteristics of regenerator and its local acoustic field are correctly chosen. According to the fact that, on one hand, amplifier acoustic gain, defined as the ration of acoustic power output (Wout) over input value (Win) which can be closed to the ratio of output over input temperature Tout/ Tin, as it may be written as:

Wout $/$ Win $=\alpha$ Tout $/$ Tin, where $\quad 0,8<\alpha<0,95$ and, Wout $=$ Win $[\alpha$ Tout $/$ Tin $]$

Moreover, Wout - Win represents the amount of heat converted into acoustic power by the heat engine (amplification) process. It thus may be written: 
Wout - Win $=\eta[1$ - Tin/Tout $]{ }^{*}$ Qwaste with $\eta \cong 70 \%$ as obtained by HEKYOM in a previous one stage engine. Here Qwaste correspond to the part of exhaust gas enthalpy that can be brought to the hot heat exchanger amplifier.

It is seen that the behaviour of each amplifier is has a strong mutual dependency. Moreover, energetic and exegetic efficiencies of each amplifier impact its required amount of heat.

Another innovative embodiment concerns the conversion of acoustic energy into electricity

Initially linear alternators were aimed to convert electricity in acoustic power and vice versa. Then an electric feedback associated with a specific starter device would be necessary in order to make the system autonomous (fig.1).

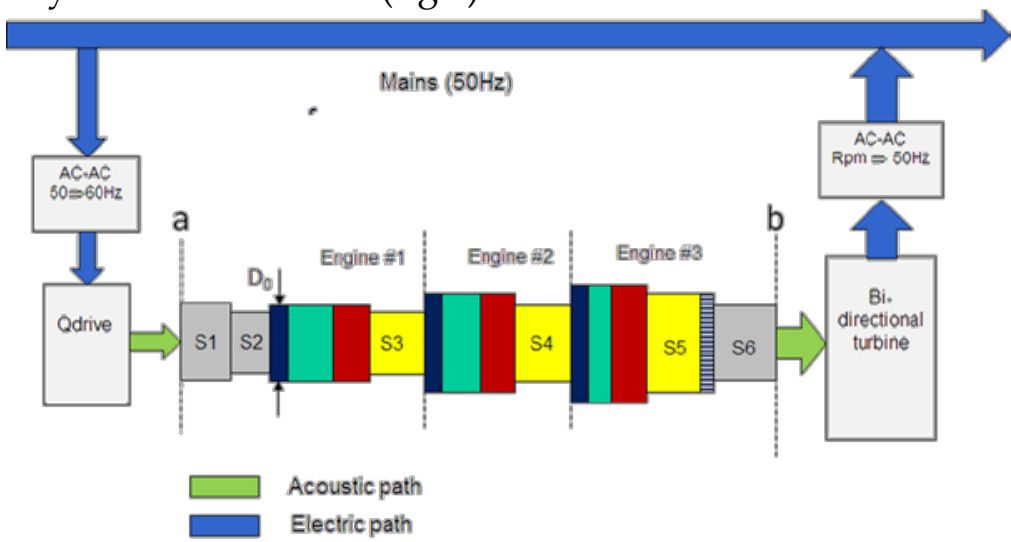

However the increasing moving mass and cost of these alternators at multi $\mathrm{kW}$ power level will hamper widespread application.

For VALTA system, it was thus decided, first to replace the electroacoustic wave generator by an acoustic feedback issue from the output acoustic wave, and second to replace the linear alternator providing conversion of acoustic energy to electric energy by a novel linear to rotation conversion of acoustic energy associated to a conventional rotating generator. (fig.2)

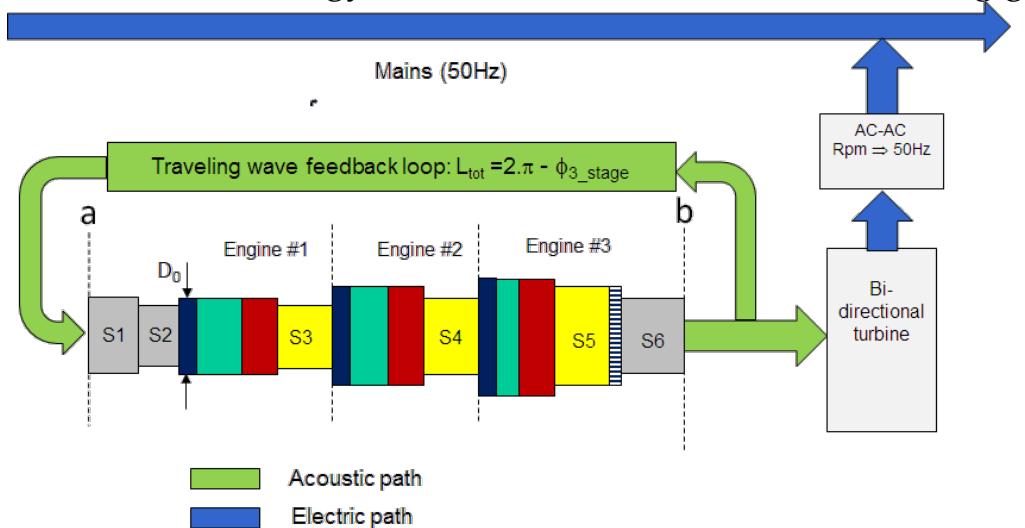

Such linear to rotation conversion of acoustic to mechanical energy system initially proposed by Aster was chosen and developed by Aster in cooperation with HEHYOM. The expected global efficiency will be of the order of $25 \%$.

The presentation will give an overview of the design choices and modifications analysed during the project and of the final design. As an example, electric or acoustic feedback has been considered and the reasons for choosing or discarding them will be mentioned.

The total VALTA budget is about 750k€. The project should be completed by the end of this year. First tests are expected in 2015 December at SDMO factory. 